\title{
Article \\ Finite Sums Involving Reciprocals of the Binomial and Central Binomial Coefficients and Harmonic Numbers
}

\author{
Necdet Batir ${ }^{1}$ and Anthony Sofo ${ }^{2, *(\mathbb{D})}$ \\ 1 Department of Mathematics, Nevşehir Hacı Bektaş Veli University, Nevşehir 50300, Turkey; \\ nbatir@nevsehir.edu.tr \\ 2 College of Engineering and Science, Victoria University, Footscray, VIC 3011, Australia \\ * Correspondence: anthony.sofo@vu.edu.au
}

check for updates

Citation: Batir, N.; Sofo, A. Finite Sums Involving Reciprocals of the Binomial and Central Binomial Coefficients and Harmonic Numbers. Symmetry 2021, 13, 2002. https:// doi.org/10.3390/sym13112002

Academic Editors: Junesang Choi and Djurdje Cvijović

Received: 16 September 2021

Accepted: 18 October 2021

Published: 22 October 2021

Publisher's Note: MDPI stays neutral with regard to jurisdictional claims in published maps and institutional affiliations.

\begin{abstract}
We prove some finite sum identities involving reciprocals of the binomial and central binomial coefficients, as well as harmonic, Fibonacci and Lucas numbers, some of which recover previously known results, while the others are new.
\end{abstract}

Keywords: binomial coefficient; central binomial coefficient; harmonic number; harmonic number sum; fibonacci numbers; lucas numbers; finite sum

JEL Classification: Primary 05A10; Secondary 05A19

\section{Introduction}

In the last hundred years or so, there has been an explosion of new results and applications of special functions in various areas of mathematics and related fields, such as engineering, quantum physics, astronomy and combinatorics. In this paper, we offer a study dealing with binomial, central binomial coefficients, and harmonic numbers that also include gamma and digamma functions and the classical Fibonacci and Lucas numbers. In 1981, Rockett [1] proved the following elegant identity: for any non-negative integer $n$,

$$
\sum_{k=0}^{n} \frac{1}{\left(\begin{array}{c}
n \\
k
\end{array}\right)}=\frac{n+1}{2^{n+1}} \sum_{k=1}^{n+1} \frac{2^{k}}{k}
$$

In fact, as stated in [2], the history of this identity goes back to 1947. This identity is entry (2.25) in Gould's collection [3], where a paper of Staver [4] is quoted. In 1997, Pla [5] rediscovered this identity and gave a different proof using generating functions. Triff [6] and Sury [7] provided new proofs of (1) using the gamma-beta integrals. Identity (1) has received great interest and motivated further combinatorial investigations. In 2002, Mansour [8] studied some finite sums involving reciprocal of the binomial coefficients and deduced a generalization of (1) together with many other results. He proved that

$$
\sum_{k=0}^{n} \frac{a^{k} b^{n-k}}{\left(\begin{array}{l}
n \\
k
\end{array}\right)}=\frac{(n+1)(a b)^{n+1}}{(a+b)^{n+2}} \sum_{k=1}^{n+1} \frac{\left(a^{k}+b^{k}\right)(a+b)^{k}}{k(a b)^{k}}
$$

In the same paper, Mansour proved the following identity:

$$
\sum_{k=0}^{n} \frac{1}{\left(\begin{array}{l}
2 n \\
2 k
\end{array}\right)}=\frac{n(2 n+1)}{2^{2 n+2}} \sum_{k=0}^{2 n+1} \frac{2^{k}}{k+1}
$$

We note that this formula is not correct, and our Example 3 gives a correct form of this identity. Note that setting $x=\frac{a}{b}$ in (2) yields 


$$
\sum_{k=0}^{n} \frac{x^{k}}{\left(\begin{array}{c}
n \\
k
\end{array}\right)}=\frac{(n+1) x^{n+1}}{(x+1)^{n+2}} \sum_{k=1}^{n+1} \frac{\left(x^{k}+1\right)(x+1)^{k}}{k x^{k}} .
$$

In 2004, Sury et al. [9] rediscovered (4). In the literature, there exist many other finite sums involving the reciprocals of the binomial coefficients. As examples, we may recall

$$
\sum_{k=0}^{n} \frac{(-1)^{k} H_{k}}{\left(\begin{array}{c}
m \\
k
\end{array}\right)}=\frac{(-1)^{n}}{\left(\begin{array}{c}
m \\
n
\end{array}\right)}\left(\frac{(n+1) H_{n}}{m+2}+\frac{m+1-n}{(m+2)^{2}}\right)-\frac{m+1}{(m+2)^{2}},
$$

where $m, n \in \mathbb{N}$ with $n \leq m$; see [10], and

$$
\sum_{k=1}^{n} \frac{(-1)^{k} H_{k}}{k\left(\begin{array}{c}
n \\
k
\end{array}\right)}=\frac{(-1)^{n} H_{n+1}}{n+1}+\sum_{k=1}^{n+1} \frac{(-1)^{k}}{k^{2}\left(\begin{array}{c}
n+1 \\
k
\end{array}\right)}
$$

see ([11] Id. 12). We note that identity (5) has been recorded as an exercise in ([12] Ex. 53, p. 315). For many interesting finite sums related to inverses of the binomial and central binomial coefficients, we refer the interested readers to the important papers [9,13-15].

For $s \in \mathbb{C}$ and $n \in \mathbb{N}$, a generalized harmonic number $H_{n}^{(s)}$ of order $s$ is defined by

$$
H_{n}^{(s)}=\sum_{k=1}^{n} \frac{1}{k^{s}}, \quad \text { and } \quad H_{n}^{(1)}=H_{n}, \quad H_{0}^{(s)}=0,
$$

where $H_{n}=1+\frac{1}{2}+\cdots+\frac{1}{n}$ are the familiar harmonic numbers. Throughout this paper, we let $\mathbb{N}_{0}=\mathbb{N} \cup\{0\}$ and $\mathbb{Z}^{-}=\{\cdots,-3,-2,-1\}$. The special binomial coefficients $\left(\begin{array}{c}2 n \\ n\end{array}\right)$ are known as the central binomial coefficients. Let $\Gamma$ be the classical gamma function of Euler, and $\psi(x)=\Gamma^{\prime}(x) / \Gamma(x)(x>0)$ be the digamma function. We may recall some basic properties of these important functions, which will be used extensively in this paper. The gamma function satisfies the reflection formula

$$
\Gamma(s) \Gamma(1-s)=\frac{\pi}{\sin (\pi s)} \quad(s \in \mathbb{C} \backslash \mathbb{Z}) .
$$

see ([16] p. 253). Another important functional equation satisfied by the gamma function is the duplication formula

$$
\Gamma\left(k+\frac{1}{2}\right)=\frac{(2 k) ! \sqrt{\pi}}{4^{k} k !} ;
$$

see ([16] p. 252). The binomial coefficients $\left(\begin{array}{c}s \\ t\end{array}\right)\left(s, t \in \mathbb{C} \backslash \mathbb{Z}^{-}\right)$can be defined in terms of the gamma function and

$$
\left(\begin{array}{l}
s \\
t
\end{array}\right)=\frac{\Gamma(s+1)}{\Gamma(t+1) \Gamma(s-t+1)} .
$$

Making use of formula (6) for $s=1 / 2-k$ and (7), we obtain

$$
\Gamma\left(\frac{1}{2}-k\right)=\frac{(-1)^{k} \Gamma(1 / 2) k ! 2^{2 k}}{(2 k) !} .
$$

We shall intensively use the following form of the binomial coefficients, which can be shown using the expressions (7)-(9).

$$
\left(\begin{array}{c}
-\frac{1}{2} \\
k
\end{array}\right)=\frac{(-1)^{k}}{4^{k}}\left(\begin{array}{c}
2 k \\
k
\end{array}\right) \text { and }\left(\begin{array}{c}
\frac{1}{2} \\
k
\end{array}\right)=\frac{(-1)^{k-1}}{4^{k}(2 k-1)}\left(\begin{array}{c}
2 k \\
k
\end{array}\right) .
$$

The following simple but useful identities will be used intensively in our ongoing analysis:

$$
\left(\begin{array}{c}
n+1 \\
k
\end{array}\right)=\frac{n+1}{k}\left(\begin{array}{c}
n \\
k-1
\end{array}\right)
$$




$$
\left(\begin{array}{c}
n+1 \\
k
\end{array}\right)=\left(\begin{array}{l}
n \\
k
\end{array}\right)+\left(\begin{array}{c}
n \\
k-1
\end{array}\right)
$$

The digamma function $\psi(x)=\frac{\Gamma^{\prime}(x)}{\Gamma(x)}(x>0)$ and harmonic numbers $H_{n}$ are related to

$$
\psi(n+1)=-\gamma+H_{n} \quad\left(n \in \mathbb{N}_{0}\right) ;
$$

see ([17] p. 31), where $\gamma=0.57721 \cdots$ is the Euler-Mascheroni constant. The digamma function $\psi$ satisfies a reflection formula and a duplication formula similar to that of the gamma function:

$$
\psi(s)-\psi(1-s)=-\pi \cot (\pi s) \quad(s \in \mathbb{C} \backslash \mathbb{Z}),
$$

and

$$
\psi\left(s+\frac{1}{2}\right)=2 \psi(2 s)-\psi(s)-2 \log 2 \quad\left(s \in \mathbb{C} \backslash \mathbb{Z}^{-}\right) ;
$$

see ([17] p. 25). Using (13)-(15), we obtain, for $k \in \mathbb{N}_{0}$,

$$
\psi\left(\frac{1}{2}-k\right)=\psi\left(\frac{1}{2}+k\right)=2 H_{2 k}-H_{k}+\psi(1 / 2) .
$$

The Fibonacci sequence $\left(F_{n}\right)_{n \geq 0}$ is given by $F_{0}=0, F_{1}=1$, and for $n \geq 2$, it satisfies the recursion relation $F_{n}=F_{n-1}+F_{n-2}$. The Lucas sequence $\left(L_{n}\right)_{n \geq 0}$ satisfies the same recursion relation with the initial values $L_{0}=2$ and $L_{1}=1$, where $n \in \mathbb{N}$. The Binet formulas for the Fibonacci numbers and Lucas numbers are given by

$$
F_{n}=\frac{\alpha^{n}-\beta^{n}}{\sqrt{5}} \text { and } L_{n}=\alpha^{n}+\beta^{n},
$$

where $\alpha=\frac{1+\sqrt{5}}{2}$ and $\beta=\frac{1-\sqrt{5}}{2}$.

The aim of this paper is to generalize identity (4), and to prove formulas (20) and (21) noted below. Differentiating our formula with respect to $s$ and $x$, and setting particular values for $s$ and $x$, we discover many interesting identities involving the reciprocals of the binomial coefficients and harmonic numbers, as well as Fibonacci and Lucas numbers. Our results also contain identities (4) and (5) and some others as special cases. We continue with the following lemma, which plays a key role in the proofs of our main results.

\section{Main Results}

Lemma 1. Let $x \in \mathbb{C} \backslash\{-1\}, n \in \mathbb{N}_{0}$, and $s \in \mathbb{C} \backslash \mathbb{Z}^{-}$. We define

$$
f_{n}(x)=\sum_{k=0}^{n} \frac{x^{k}}{\left(\begin{array}{c}
n+s \\
k
\end{array}\right)} .
$$

Then, $f_{n}$ satisfies

$$
f_{n+1}(x)=\frac{x}{x+1} \cdot \frac{n+s+2}{n+s+1} \cdot f_{n}(x)+\frac{x^{n+2}}{(x+1)\left(\begin{array}{c}
n+s+1 \\
n+1
\end{array}\right)}+\frac{1}{x+1} .
$$

Proof. We, clearly, have

$$
\begin{aligned}
f_{n+1}(x)-f_{n}(x) & =\sum_{k=0}^{n+1} \frac{x^{k}}{\left(\begin{array}{c}
n+s+1 \\
k
\end{array}\right)}-\sum_{k=0}^{n} \frac{x^{k}}{\left(\begin{array}{c}
n+s \\
k
\end{array}\right)} \\
& =\sum_{k=0}^{n}\left(\frac{1}{\left(\begin{array}{c}
n+s+1 \\
k
\end{array}\right)}-\frac{1}{\left(\begin{array}{c}
n+s \\
k
\end{array}\right)}\right) x^{k}+\frac{x^{n+1}}{\left(\begin{array}{c}
n+s+1 \\
n+1
\end{array}\right)} .
\end{aligned}
$$


By (11) and (12), we can easily show that

$$
\frac{1}{\left(\begin{array}{c}
n+s+1 \\
k
\end{array}\right)}-\frac{1}{\left(\begin{array}{c}
n+s \\
k
\end{array}\right)}=-\frac{k}{(n+s+1)\left(\begin{array}{c}
n+s \\
k
\end{array}\right)} .
$$

Hence,

$$
\sum_{k=1}^{n+1} \frac{x^{k}}{\left(\begin{array}{c}
n+s+1 \\
k
\end{array}\right)}-\sum_{k=1}^{n} \frac{x^{k}}{\left(\begin{array}{c}
n+s \\
k
\end{array}\right)}=-\sum_{k=1}^{n} \frac{k x^{k}}{(n+s+1)\left(\begin{array}{c}
n+s \\
k
\end{array}\right)}+\frac{x^{n+1}}{\left(\begin{array}{c}
n+s+1 \\
n+1
\end{array}\right)} .
$$

Dividing both sides of this equation by $x$ and then integrating over the interval $[0, u]$ and replacing $u=x$, we obtain

$$
\sum_{k=1}^{n+1} \frac{x^{k}}{k\left(\begin{array}{c}
n+s+1 \\
k
\end{array}\right)}-\sum_{k=1}^{n} \frac{x^{k}}{k\left(\begin{array}{c}
n+s \\
k
\end{array}\right)}=\frac{x^{n+1}}{(n+1)\left(\begin{array}{c}
n+s+1 \\
n+1
\end{array}\right)}-\sum_{k=1}^{n} \frac{x^{k}}{(n+s+1)\left(\begin{array}{c}
n+s \\
k
\end{array}\right)} .
$$

By (11), this can be written as follows:

$$
\begin{aligned}
\frac{1}{n+s+1} \sum_{k=1}^{n+1} \frac{x^{k}}{\left(\begin{array}{c}
n+s \\
k-1
\end{array}\right)}-\frac{1}{n+s} \sum_{k=1}^{n} \frac{x^{k}}{\left(\begin{array}{c}
n+s-1 \\
k-1
\end{array}\right)} & =-\frac{1}{n+s+1} \sum_{k=1}^{n} \frac{x^{k}}{\left(\begin{array}{c}
n+s \\
k
\end{array}\right)} \\
& +\frac{x^{n+1}}{(n+s+1)\left(\begin{array}{c}
n+s \\
n
\end{array}\right)} .
\end{aligned}
$$

Letting $k-1=k^{\prime}$ in the sums on the left-hand side, and then dropping the prime, we obtain, after a short computation,

$$
\begin{aligned}
\frac{x}{n+s+1} \sum_{k=0}^{n} \frac{x^{k}}{\left(\begin{array}{c}
n+s \\
k
\end{array}\right)}-\frac{x}{n+s} \sum_{k=0}^{n-1} \frac{x^{k}}{\left(\begin{array}{c}
n+s-1 \\
k
\end{array}\right)} & =-\frac{1}{n+s+1} \sum_{k=1}^{n} \frac{x^{k}}{\left(\begin{array}{c}
n+s \\
k
\end{array}\right)} \\
& +\frac{x^{n+1}}{(n+s+1)\left(\begin{array}{c}
n+s \\
n
\end{array}\right)}
\end{aligned}
$$

or

$$
\frac{(x+1) f_{n}(x)}{n+s+1}-\frac{x f_{n-1}(x)}{n+s}=\frac{1}{n+s+1}+\frac{x^{n+1}}{(n+s+1)\left(\begin{array}{c}
n+s \\
n
\end{array}\right)} .
$$

If we replace $n$ by $n+1$ and take $x \neq-1$, we see that this is equivalent to (17).

Setting $x=-1$ in (19), and replacing $n$ by $n+1$, we obtain

Corollary 1. Let $s \in \mathbb{C} \backslash \mathbb{Z}^{-}$, and $n \in \mathbb{N}_{0}$. Then,

$$
\sum_{k=0}^{n} \frac{(-1)^{k}}{\left(\begin{array}{c}
n+s \\
k
\end{array}\right)}=\frac{n+s+1}{n+s+2}\left(\frac{(-1)^{n}}{\left(\begin{array}{c}
n+s+1 \\
n+1
\end{array}\right)}+1\right) .
$$

In particular, replacing s by $s-n$ here yields for $s \in \mathbb{C} \backslash \mathbb{Z}^{-}$and $n \leq s$ if $s \in \mathbb{N}_{0}$

$$
\sum_{k=0}^{n} \frac{(-1)^{k}}{\left(\begin{array}{l}
s \\
k
\end{array}\right)}=\frac{s+1}{s+2}\left(\frac{(-1)^{n}}{\left(\begin{array}{l}
s+1 \\
n+1
\end{array}\right)}+1\right)
$$

The main theorem now follows. 
Theorem 1. Let $n \in \mathbb{N}_{0}, s \in \mathbb{C} \backslash \mathbb{Z}^{-}$, and $x \in \mathbb{C} \backslash\{-1\}$. Then,

$$
\begin{aligned}
\sum_{k=0}^{n} \frac{x^{k}}{\left(\begin{array}{c}
n+s \\
k
\end{array}\right)} & =(n+s+1)\left(\frac{x}{x+1}\right)^{n}\left(\frac{x}{x+1} \sum_{k=0}^{n} \frac{(x+1)^{k}}{(k+1)\left(\begin{array}{c}
k+s+1 \\
k+1
\end{array}\right)}\right. \\
& \left.+\frac{1}{x+1} \sum_{k=0}^{n} \frac{1}{k+s+1}\left(\frac{x+1}{x}\right)^{k}\right) .
\end{aligned}
$$

Proof. We prove by mathematical induction. Clearly, (21) is true for $n=0,1$. We assume that it is also true for $n$. So, by the inductive hypothesis and Lemma 1, we have

$$
\begin{aligned}
& f_{n+1}(x)=\frac{x}{x+1} \frac{n+s+2}{n+s+1}\left((n+s+1)\left(\frac{x}{x+1}\right)^{n}\right. \\
& \left.\times\left(\frac{x}{x+1} \sum_{k=0}^{n} \frac{(x+1)^{k}}{(k+1)\left(\begin{array}{c}
k+s+1 \\
k+1
\end{array}\right)}+\frac{1}{x+1} \sum_{k=0}^{n} \frac{1}{k+s+1}\left(\frac{x+1}{x}\right)^{k}\right)\right) \\
& +\frac{x^{n+2}}{(x+1)\left(\begin{array}{c}
n+s+1 \\
n+1
\end{array}\right)}+\frac{1}{x+1} .
\end{aligned}
$$

Simplifying this identity leads to

$$
\begin{aligned}
f_{n+1}(x) & =\left(\frac{x}{x+1}\right)^{n+1}(n+s+2)\left(\frac{x}{x+1} \sum_{k=0}^{n} \frac{(x+1)^{k}}{(k+1)\left(\begin{array}{c}
k+s+1 \\
k+1
\end{array}\right)}\right. \\
& \left.+\frac{1}{x+1} \sum_{k=0}^{n} \frac{1}{k+s+1}\left(\frac{x+1}{x}\right)^{k}\right)+\frac{x^{n+2}}{(x+1)\left(\begin{array}{c}
n+s+1 \\
n+1
\end{array}\right)}+\frac{1}{x+1} .
\end{aligned}
$$

Some algebraic computations lead to

$$
\begin{aligned}
f_{n+1}(x) & =(n+s+2)\left(\frac{x}{x+1}\right)^{n+1}\left(\frac{x}{x+1} \sum_{k=0}^{n+1} \frac{(x+1)^{k}}{(k+1)\left(\begin{array}{c}
k+s+1 \\
k+1
\end{array}\right)}\right. \\
& +\frac{1}{x+1} \sum_{k=0}^{n+1} \frac{1}{k+s+1}\left(\frac{x+1}{x}\right)^{k}-\frac{x}{x+1} \frac{(x+1)^{n+1}}{(n+s+2)\left(\begin{array}{c}
n+s+1 \\
n+1
\end{array}\right)} \\
& \left.-\frac{1}{x+1} \frac{1}{n+s+2}\left(\frac{x+1}{x}\right)^{k}\right)+\frac{x^{n+2}}{(x+1)\left(\begin{array}{c}
n+s+1 \\
n+1
\end{array}\right)}+\frac{1}{x+1} .
\end{aligned}
$$

This can be simplified to

$$
\begin{aligned}
f_{n+1}(x) & =(n+s+2)\left(\frac{x}{x+1}\right)^{n+1}\left(\frac{x}{x+1} \sum_{k=0}^{n+1} \frac{(x+1)^{k}}{(k+1)\left(\begin{array}{c}
k+s+1 \\
k+1
\end{array}\right)}\right. \\
& \left.+\frac{1}{x+1} \sum_{k=0}^{n+1} \frac{1}{k+s+1}\left(\frac{x+1}{x}\right)^{k}\right)-\left(\frac{x}{x+1}\right)^{n+2} \frac{(x+1)^{n+1}}{\left(\begin{array}{c}
n+s+1 \\
n+1
\end{array}\right)} \\
& -\left(\frac{x}{x+1}\right)^{n+1} \frac{1}{x+1}\left(\frac{x+1}{x}\right)^{n+1}+\frac{x^{n+2}}{(x+1)\left(\begin{array}{c}
n+s+1 \\
n+1
\end{array}\right)}+\frac{1}{x+1}
\end{aligned}
$$

or

$$
\begin{aligned}
f_{n+1}(x) & =(n+s+2)\left(\frac{x}{x+1}\right)^{n+1}\left(\frac{x}{x+1} \sum_{k=0}^{n+1} \frac{(x+1)^{k}}{(k+1)\left(\begin{array}{c}
k+s+1 \\
k+1
\end{array}\right)}\right. \\
& \left.+\frac{1}{x+1} \sum_{k=0}^{n+1} \frac{1}{k+s+1}\left(\frac{x+1}{x}\right)^{k}\right) .
\end{aligned}
$$


This reveals that (21) is valid for $n+1$, and the proof is complete.

Corollary 2. Setting $x=1$ in (21), we obtain, for $n \in \mathbb{N}_{0}$ and $s \in \mathbb{C} \backslash \mathbb{Z}^{-}$,

$$
\sum_{k=0}^{n} \frac{1}{\left(\begin{array}{c}
s+n \\
k
\end{array}\right)}=\frac{n+s+1}{2^{n+1}}\left(\sum_{k=0}^{n} \frac{2^{k}}{(k+1)\left(\begin{array}{c}
s+k+1 \\
k+1
\end{array}\right)}+\sum_{k=0}^{n} \frac{2^{k}}{k+s+1}\right) .
$$

Corollary 3. Setting $s=0$ in (22), we obtain, for $x \neq-1$,

$$
\begin{aligned}
\sum_{k=0}^{n} \frac{x^{k}}{\left(\begin{array}{l}
n \\
k
\end{array}\right)} & =(n+1)\left(\frac{x}{x+1}\right)^{n}\left(\frac{x}{x+1} \sum_{k=0}^{n} \frac{(x+1)^{k}}{k+1}\right. \\
& \left.+\frac{1}{x+1} \sum_{k=0}^{n} \frac{1}{k+1}\left(\frac{x+1}{x}\right)^{k}\right),
\end{aligned}
$$

which is equivalent to (4).

Corollary 4. Setting $s=-\frac{1}{2}$ in (21), we obtain, for $n \in \mathbb{N}_{0}$ and $x \in \mathbb{C} \backslash\{-1,0\}$,

$$
\begin{aligned}
\sum_{k=0}^{n} \frac{\left(\begin{array}{c}
2 k \\
k
\end{array}\right)}{(4 x)^{k}\left(\begin{array}{c}
n \\
k
\end{array}\right)} & =\frac{(2 n+1)\left(\begin{array}{c}
2 n \\
n
\end{array}\right) x}{4^{n}(x+1)^{n+1}}\left(\sum_{k=0}^{n} \frac{4^{k}(x+1)^{k}}{(2 k+1)\left(\begin{array}{c}
2 k \\
k
\end{array}\right)}\right. \\
& \left.+\frac{1}{x} \sum_{k=0}^{n} \frac{1}{2 k+1}\left(\frac{x+1}{x}\right)^{k}\right) .
\end{aligned}
$$

\section{Examples}

Example 1. Setting $x=1$ in (23), we obtain

$$
\sum_{k=0}^{n} \frac{1}{\left(\begin{array}{l}
n \\
k
\end{array}\right)}=\frac{n+1}{2^{n}} \sum_{k=0}^{n} \frac{2^{k}}{k+1}
$$

Example 2. Setting $s=0$ in (20) and replacing $n$ by $2 n$, we obtain

$$
\sum_{k=0}^{n} \frac{(-1)^{k}}{\left(\begin{array}{l}
n \\
k
\end{array}\right)}=\frac{(n+1)\left(1+(-1)^{n}\right)}{n+2}
$$

Summing the Equations (24) and (25) side by side and replacing $n$ by $2 n$, one obtains the following identity, which recovers (3).

Example 3. For $n \in \mathbb{N}_{0}$, we have

$$
\sum_{k=0}^{n} \frac{1}{\left(\begin{array}{l}
2 n \\
2 k
\end{array}\right)}=\frac{2 n+1}{2^{2 n+1}} \sum_{k=0}^{2 n+1} \frac{2^{k}}{k+1}
$$

Example 4. For $x \in \mathbb{C} \backslash\{-1,1\}$, we have

$$
\begin{aligned}
\sum_{k=0}^{n} \frac{x^{2 k}}{\left(\begin{array}{l}
2 n \\
2 k
\end{array}\right)} & =\frac{(2 n+1) x^{2 n}}{2}\left(x \sum_{k=0}^{2 n} \frac{(x+1)^{k-2 n-1}-(1-x)^{k-2 n-1}}{(k+1)}\right. \\
& \left.+\sum_{k=0}^{2 n} \frac{(x+1)^{k-2 n-1}-(x-1)^{k-2 n-1}}{(k+1) x^{k}}\right) .
\end{aligned}
$$


Proof. Replacing $x$ by $-x$ in (23), we obtain, for $x \neq 1$,

$$
\begin{aligned}
\sum_{k=0}^{n} \frac{(-1)^{k} x^{k}}{\left(\begin{array}{l}
n \\
k
\end{array}\right)} & =(n+1)\left(\frac{x}{x-1}\right)^{n}\left(\frac{x}{x-1} \sum_{k=0}^{n} \frac{(1-x)^{k}}{k+1}\right. \\
& \left.+\frac{1}{1-x} \sum_{k=0}^{n} \frac{1}{k+1}\left(\frac{x-1}{x}\right)^{k}\right) .
\end{aligned}
$$

If we replace $n$ by $2 n$ and sum the Equations (27) and (28) side by side, the result follows after a simple computation.

Example 5. For $n \in \mathbb{N}_{0}$, we have

$$
\begin{aligned}
\sum_{k=0}^{n} \frac{(-1)^{k}}{\left(\begin{array}{l}
2 n \\
2 k
\end{array}\right)} & =\frac{\left(1+(-1)^{n}\right)(2 n+1)}{2^{n}} \sum_{k=0}^{2 n} \frac{(\sqrt{2})^{k-1} \sin \left(\frac{(2 n-k+1) \pi}{4}\right)}{k+1} \\
& =\frac{\left(1+(-1)^{n}\right)(2 n+1)}{2}\left(\sum_{k=0}^{\left\lfloor\frac{n}{2}\right\rfloor} \frac{(-1)^{k}}{4^{k}(2 n-4 k+1)}\right. \\
& \left.-2 \sum_{k=1}^{\left\lfloor\frac{n+1}{2}\right\rfloor} \frac{(-1)^{k}}{4^{k}(2 n-4 k+3)}+\sum_{k=0}^{\left\lfloor\frac{n-1}{2}\right\rfloor} \frac{(-1)^{k}}{4^{k}(2 n-4 k)}\right)
\end{aligned}
$$

where $\lfloor\cdot\rfloor$ is the greatest integer function.

Proof. Setting $x=i \in \mathbb{C}$ in (27), we obtain

$$
\begin{aligned}
\sum_{k=0}^{n} \frac{(-1)^{k}}{\left(\begin{array}{l}
2 n \\
2 k
\end{array}\right)} & =\frac{(-1)^{n}(2 n+1)}{2}\left(i \sum_{k=0}^{2 n} \frac{(1+i)^{k-2 n-1}-(1-i)^{k-2 n-1}}{k+1}\right. \\
& \left.+\sum_{k=0}^{2 n} \frac{(1+i)^{k-2 n-1}-(i-1)^{k-2 n-1}}{(k+1) i^{k}}\right)
\end{aligned}
$$

An easy computation shows that

$$
\frac{(1+i)^{k-2 n-1}-(i-1)^{k-2 n-1}}{i^{k}}=(-1)^{n} i\left((1+i)^{k-2 n-1}-(1-i)^{k-2 n-1}\right) .
$$

Thus, we have

$$
\sum_{k=0}^{n} \frac{(-1)^{k}}{\left(\begin{array}{l}
2 n \\
2 k
\end{array}\right)}=\left(1+(-1)^{n}\right)(2 n+1) i \sum_{k=0}^{2 n} \frac{(1+i)^{k-2 n-1}-(1-i)^{k-2 n-1}}{k+1} .
$$

The proof of (29) follows from

$$
i\left((1+i)^{k-2 n-1}-(1-i)^{k-2 n-1}\right)=2(\sqrt{2})^{k-2 n-1} \sin \left(\frac{(2 n-k+1) \pi}{4}\right) .
$$

After some computations, we see that

$$
\begin{aligned}
& i \sum_{k=0}^{2 n} \frac{(1+i)^{k-2 n-1}-(1-i)^{k-2 n-1}}{k+1}=\sum_{k=0}^{\left\lfloor\frac{n}{2}\right\rfloor} \frac{(-1)^{k}}{2^{2 k}(2 n-4 k+1)} \\
& -2 \sum_{k=1}^{\left\lfloor\frac{n+1}{2}\right\rfloor} \frac{(-1)^{k}}{2^{2 k}(2 n-4 k+3)}+\sum_{k=0}^{\left\lfloor\frac{n-1}{2}\right\rfloor} \frac{(-1)^{k}}{2^{2 k}(2 n-4 k)}
\end{aligned}
$$

Hence, from (32) and (33), we see that (30) is valid. 
Remark 1. From (30), we conclude

$$
\sum_{k=0}^{2 n+1} \frac{(-1)^{k}}{\left(\begin{array}{c}
4 n+2 \\
2 k
\end{array}\right)}=0
$$

and

$$
\sum_{k=1}^{2 n} \frac{(-1)^{k}}{\left(\begin{array}{c}
4 n \\
2 k
\end{array}\right)}=\frac{(-1)^{n}(4 n+1)}{4^{n}} \sum_{k=0}^{n-1}\left(\frac{1}{4 k+1}-\frac{2}{4 k+3}-\frac{1}{k+1}\right)(-4)^{k}
$$

Example 6. For all $x \in \mathbb{C}$ with $x \neq-1$, and all non-negative integers $n$, we have

$$
\begin{aligned}
\sum_{k=0}^{n} \frac{(-4 x)^{k}}{\left(\begin{array}{c}
2 k \\
k
\end{array}\right)} & =\frac{x}{x+1} \cdot \frac{(-4 x)^{n}}{\left(\begin{array}{c}
2 n \\
n
\end{array}\right)} \sum_{k=0}^{n} \frac{(-1)^{k+1}\left(\begin{array}{c}
2 k \\
k
\end{array}\right)}{4^{k}(2 k-1)(x+1)^{k}\left(\begin{array}{c}
n \\
k
\end{array}\right)} \\
& -\frac{1}{x+1} \sum_{k=0}^{n} \frac{1}{2 k-1}\left(\frac{x}{x+1}\right)^{k} .
\end{aligned}
$$

Proof. Setting $s=-\frac{1}{2}-n$ in (21), we obtain

$$
\begin{aligned}
\sum_{k=0}^{n} \frac{x^{k}}{\left(\begin{array}{c}
-\frac{1}{2} \\
k
\end{array}\right)} & =\frac{1}{2}\left(\frac{x}{x+1}\right)^{n+1} \sum_{k=0}^{n} \frac{(x+1)^{k}}{(k+1)\left(\begin{array}{c}
\frac{1}{2}-n+k \\
k+1
\end{array}\right)} \\
& +\frac{x^{n}}{(x+1)^{n+1}} \sum_{k=0}^{n} \frac{1}{1-2 n+2 k}\left(\frac{x+1}{x}\right)^{k} .
\end{aligned}
$$

Applying the first identity in (10) and

$$
(k+1)\left(\begin{array}{c}
\frac{1}{2}-n+k \\
k+1
\end{array}\right)=\frac{\left(\frac{1}{2}-n+k\right)(-1)^{k}\left(\begin{array}{c}
2 n \\
n
\end{array}\right)\left(\begin{array}{c}
n \\
k
\end{array}\right)}{4^{k}\left(\begin{array}{c}
2 n-2 k \\
n-k
\end{array}\right)},
$$

we obtain from (35)

$$
\begin{aligned}
\sum_{k=0}^{n} \frac{(-4 x)^{k}}{\left(\begin{array}{c}
2 k \\
k
\end{array}\right)} & =\frac{1}{\left(\begin{array}{c}
2 n \\
n
\end{array}\right)}\left(\frac{x}{x+1}\right)^{n+1} \sum_{k=0}^{n} \frac{(-1)^{k} 4^{k}(x+1)^{k}\left(\begin{array}{c}
2 n-2 k \\
n-k
\end{array}\right)}{(1-2 n+2 k)\left(\begin{array}{c}
n \\
k
\end{array}\right)} \\
& -\frac{1}{x+1}\left(\frac{x}{x+1}\right)^{n} \sum_{k=0}^{n} \frac{1}{2 n-2 k-1}\left(\frac{x+1}{x}\right)^{k} .
\end{aligned}
$$

Changing the index $k$ to $n-k$ in the sums on the right-hand side yields the desired result.

Remark 2. Applying the operator $\left(x \frac{d}{d x}\right)^{m}$ to both sides of (34) enables us to find formulas for the family of sums

$$
\sum_{k=0}^{n} \frac{(-1)^{k} 4^{k} k^{m}}{\left(\begin{array}{c}
2 k \\
k
\end{array}\right)}
$$

Example 7. Setting $x=1$ in (34), we obtain

$$
\sum_{k=0}^{n} \frac{(-4)^{k}}{\left(\begin{array}{c}
2 k \\
k
\end{array}\right)}=\frac{(-4)^{n}}{2\left(\begin{array}{c}
2 n \\
n
\end{array}\right)} \sum_{k=0}^{n} \frac{(-1)^{k+1}\left(\begin{array}{c}
2 k \\
k
\end{array}\right)}{8^{k}(2 k-1)\left(\begin{array}{c}
n \\
k
\end{array}\right)}-\frac{1}{2} \sum_{k=0}^{n} \frac{1}{2^{k}(2 k-1)}
$$


Example 8. Setting $x=\frac{1}{4}$ in (34), we obtain

$$
\sum_{k=0}^{n} \frac{(-1)^{k}}{\left(\begin{array}{c}
2 k \\
k
\end{array}\right)}=\frac{(-1)^{n}}{5\left(\begin{array}{c}
2 n \\
n
\end{array}\right)} \sum_{k=0}^{n} \frac{(-1)^{k+1}\left(\begin{array}{c}
2 k \\
k
\end{array}\right)}{5^{k}(2 k-1)\left(\begin{array}{c}
n \\
k
\end{array}\right)}-\frac{4}{5} \sum_{k=0}^{n} \frac{1}{5^{k}(2 k-1)}
$$

Example 9. Setting $x=-\frac{1}{4}$ in (34), we obtain

$$
\sum_{k=0}^{n} \frac{1}{\left(\begin{array}{c}
2 k \\
k
\end{array}\right)}=\frac{1}{3\left(\begin{array}{c}
2 n \\
n
\end{array}\right)} \sum_{k=0}^{n} \frac{(-1)^{k}\left(\begin{array}{c}
2 k \\
k
\end{array}\right)}{3^{k}(2 k-1)\left(\begin{array}{c}
n \\
k
\end{array}\right)}-\frac{4}{3} \sum_{k=0}^{n} \frac{(-1)^{k}}{3^{k}(2 k-1)} .
$$

Example 10. For all $s \in \mathbb{C}$, which is not a negative integer, and $n \in \mathbb{N}_{0}$, we have

$$
\begin{aligned}
& \sum_{k=0}^{n} \frac{(-1)^{k+1}(\psi(n+s+1)-\psi(n+s-k+1))}{\left(\begin{array}{c}
n+s \\
k
\end{array}\right)} \\
& =\frac{1}{(n+s+2)^{2}}+\frac{(-1)^{n}}{(n+s+2)^{2}\left(\begin{array}{c}
n+s+1 \\
n+1
\end{array}\right)} \\
& -\frac{n+s+1}{n+s+2} \cdot \frac{(-1)^{n}(\psi(n+s+2)-\psi(s+1))}{\left(\begin{array}{c}
n+s+1 \\
n+1
\end{array}\right)} .
\end{aligned}
$$

Proof. The proof follows from (20) by differentiating both sides with respect to $s$.

Example 11. Setting $s=-\frac{1}{2}-n$ in (36), we obtain by (13)

$$
\sum_{k=0}^{n} \frac{4^{k}\left(2 H_{2 k}-H_{k}\right)}{\left(\begin{array}{c}
2 k \\
k
\end{array}\right)}=\frac{4}{9}+\frac{2(n+1) 4^{n}\left(6 H_{2 n}-3 H_{n}-2\right)}{9\left(\begin{array}{c}
2 n \\
n
\end{array}\right)}
$$

Remark 3. The odd harmonic numbers $O_{n}=1+\frac{1}{3}+\frac{1}{5}+\cdots+\frac{1}{2 n-1}$ and the harmonic numbers are connected with $\mathrm{O}_{n}=H_{2 n}-\frac{1}{2} H_{n}$. Therefore, Example 11 can be rewritten in the following elegant form:

$$
\sum_{k=1}^{n} \frac{4^{k} O_{k}}{\left(\begin{array}{c}
2 k \\
k
\end{array}\right)}=\frac{2}{9}+\frac{2(n+1) 4^{n}\left(3 O_{n}-1\right)}{9\left(\begin{array}{c}
2 n \\
n
\end{array}\right)} .
$$

This formula, it is acknowledged, is included in the preprint [18], sent as a private communication to the first author. Some other harmonic number identities may be seen in [19-21].

Example 12. For all non-negative integers $n$, we have

$$
\sum_{k=0}^{n} \frac{(-1)^{k} H_{k}}{\left(\begin{array}{l}
n \\
k
\end{array}\right)}=\frac{(-1)^{n}(n+1) H_{n}-1}{n+2}+\frac{1+(-1)^{n}}{(n+2)^{2}}
$$

Proof. If we let $s=0$ in (36), we obtain

$$
\begin{aligned}
& \sum_{k=0}^{n} \frac{(-1)^{k+1}(\psi(n+1)-\psi(n-k+1))}{\left(\begin{array}{l}
n \\
k
\end{array}\right)}=\frac{1+(-1)^{n}}{(n+2)^{2}} \\
& -\frac{n+1}{n+2} \cdot \frac{(-1)^{n}(\psi(n+2)-\psi(1))}{\left(\begin{array}{l}
n+1 \\
n+1
\end{array}\right)} .
\end{aligned}
$$

From the relation $\psi(n+1)=\psi(1)+H_{n}$, it follows that

$$
H_{n} \sum_{k=0}^{n} \frac{(-1)^{k+1}}{\left(\begin{array}{l}
n \\
k
\end{array}\right)}+\sum_{k=0}^{n} \frac{(-1)^{k} H_{n-k}}{\left(\begin{array}{l}
n \\
k
\end{array}\right)}=\frac{1+(-1)^{n}}{(n+2)^{2}}-\frac{(-1)^{n}(n+1) H_{n+1}}{n+2} .
$$

Using (25) and changing the index $k$ to $n-k$ in the second sum, the conclusion follows. 
Remark 4. Formula (37) corrects the corresponding identity in [22] (p. 2).

Example 13. For all non-negative integers $n$, we have

$$
\sum_{k=0}^{n} \frac{H_{k}}{\left(\begin{array}{l}
n \\
k
\end{array}\right)}=\frac{1+(n+1) H_{n}}{2^{n+1}} \sum_{k=1}^{n+1} \frac{2^{k}}{k}-\frac{n+1}{2^{n+2}} \sum_{k=1}^{n+1} \frac{2^{k} H_{k}}{k}-\frac{n+1}{2^{n+2}} \sum_{k=1}^{n+1} \frac{2^{k}}{k^{2}} .
$$

Proof. Differentiating both sides of (21) with respect to $s$, we obtain

$$
\begin{aligned}
& \sum_{k=0}^{n} \frac{(\psi(s+n-k+1)-\psi(s+n+1)) x^{k}}{\left(\begin{array}{c}
n+s \\
k
\end{array}\right)}=\frac{x^{n}}{(x+1)^{n+1}} \sum_{k=0}^{n} \frac{\left(\frac{x+1}{x}\right)^{k}}{s+k+1} \\
& +\left(\frac{x}{x+1}\right)^{n+1} \sum_{k=0}^{n} \frac{(x+1)^{k}}{(k+1)\left(\begin{array}{c}
s+k+1 \\
k+1
\end{array}\right)}-\frac{(s+n+1) x^{n}}{(x+1)^{n+1}} \sum_{k=0}^{n} \frac{\left(\frac{x+1}{x}\right)^{k}}{(s+k+1)^{2}} \\
& +(s+n+1)\left(\frac{x}{x+1}\right)^{n+1} \sum_{k=0}^{n} \frac{(\psi(s+1)-\psi(s+k+2))(x+1)^{k}}{(k+1)\left(\begin{array}{c}
s+k+1 \\
k+1
\end{array}\right)} .
\end{aligned}
$$

Putting $s=0$ and $x=1$ in (39) and using (13), we find

$$
\sum_{k=0}^{n} \frac{H_{k}}{\left(\begin{array}{l}
n \\
k
\end{array}\right)}=\sum_{k=0}^{n} \frac{H_{n}}{\left(\begin{array}{l}
n \\
k
\end{array}\right)}+\frac{1}{2^{n+1}} \sum_{k=1}^{n+1} \frac{2^{k}}{k}-\frac{n+1}{2^{n+2}} \sum_{k=1}^{n+1} \frac{2^{k} H_{k}}{k}-\frac{n+1}{2^{n+2}} \sum_{k=1}^{n} \frac{2^{k}}{k^{2}} .
$$

Now, the proof follows from (24).

Example 14. Setting $s=-\frac{1}{2}-n$ in (20), we obtain, for all non-negative integers $n$

$$
\sum_{k=0}^{n} \frac{4^{k}}{\left(\begin{array}{c}
2 k \\
k
\end{array}\right)}=\frac{2(n+1) 4^{n}}{3\left(\begin{array}{c}
2 n \\
n
\end{array}\right)}+\frac{1}{3}
$$

Remark 5. Identity 14 is not new and can be found in [14] (Equation (1.6)).

Example 15. Setting $s=\frac{1}{2}-n$ in (20), we obtain by (10) for $n \in \mathbb{N}_{0}$

$$
\sum_{k=0}^{n} \frac{(2 k-1) 4^{k}}{\left(\begin{array}{c}
2 k \\
k
\end{array}\right)}=\frac{2^{2 n+1}(n+1)(2 n-1)}{5\left(\begin{array}{c}
2 n \\
n
\end{array}\right)}-\frac{3}{5}
$$

Example 16. Repeated differentiation of both sides of (20) with respect to s gives

$$
\begin{aligned}
& \sum_{k=0}^{n}(-1)^{k}\left(\frac{(\psi(s+n+1)-\psi(s+n-k+1))^{2}}{\left(\begin{array}{c}
n+s \\
k
\end{array}\right)}\right. \\
& \left.-\frac{\psi^{\prime}(s+n+1)-\psi^{\prime}(s+n-k+1)}{\left(\begin{array}{c}
n+s \\
k
\end{array}\right)}\right)=-\frac{2}{(n+s+2)^{3}} \\
& -\frac{2(-1)^{n}}{(n+s+2)^{3}\left(\begin{array}{c}
n+s+1 \\
n+1
\end{array}\right)}-\frac{2(-1)^{n}(\psi(s+n+2)-\psi(s+1))}{(n+s+2)^{2}\left(\begin{array}{c}
n+s+1 \\
n+1
\end{array}\right)} \\
& +\frac{(-1)^{n}(n+s+1)}{(n+s+2)\left(\begin{array}{c}
n+s+1 \\
n+1
\end{array}\right)}\left((\psi(s+n+2)-\psi(s+1))^{2}\right. \\
& \left.-\left(\psi^{\prime}(n+s+2)-\psi^{\prime}(s+1)\right)\right) .
\end{aligned}
$$


Example 17. Let $n \in \mathbb{N}_{0}$. Then, we have

$$
\begin{aligned}
\sum_{k=0}^{n} \frac{(-1)^{k}\left(H_{k}^{2}-H_{k}^{(2)}\right)}{\left(\begin{array}{l}
n \\
k
\end{array}\right)} & =\frac{(-1)^{n}(n+1)\left(H_{n}^{2}-H_{n}^{(2)}\right)}{n+2} \\
& +\frac{2\left(1+(-1)^{n} H_{n}\right)}{(n+2)^{2}}-\frac{2\left(1+(-1)^{n}\right)}{(n+2)^{3}} .
\end{aligned}
$$

Proof. The proof follows from (42) by setting $s=0$, and using the relations $\psi(n+1)=$ $\psi(1)+H_{n}$ and $\psi^{\prime}(1)-\psi^{\prime}(n+1)=H_{n}^{(2)}$.

Example 18. Setting $s=\frac{1}{2}$ in (20), we obtain

$$
\sum_{k=0}^{n} \frac{(-1)^{k}(2 k+1)\left(\begin{array}{c}
2 k \\
k
\end{array}\right)}{4^{k}\left(\begin{array}{c}
n \\
k
\end{array}\right)}=\frac{2(n+1)}{2 n+5}\left(1+\frac{(-1)^{n}(2 n+1)(2 n+3)\left(\begin{array}{c}
2 n \\
n
\end{array}\right)}{2(n+1) 4^{n}}\right) .
$$

Example 19. Differentiating both sides of (21) with respect to $x$, and then setting $x=1$, we obtain

$$
\sum_{k=1}^{n} \frac{k}{\left(\begin{array}{c}
s+n \\
k
\end{array}\right)}=\frac{n+s+1}{2^{n+2}}\left(\sum_{k=0}^{n} \frac{(n+k+1) 2^{k}}{(k+1)\left(\begin{array}{c}
s+k+1 \\
k+1
\end{array}\right)}+\sum_{k=0}^{n} \frac{(n-k-1) 2^{k}}{s+k+1}\right) .
$$

In particular, for $s=0$, the result is

$$
\sum_{k=1}^{n} \frac{k}{\left(\begin{array}{l}
n \\
k
\end{array}\right)}=\frac{n(n+1)}{2^{n+1}} \sum_{k=0}^{n} \frac{2^{k}}{k+1}
$$

Remark 6. In $[23,24]$, the authors considered the alternating finite sums

$$
\sum_{k=0}^{n} \frac{(-1)^{k} k^{m}}{\left(\begin{array}{l}
n \\
k
\end{array}\right)}
$$

and they provided many properties of them involving closed form evaluations. If we apply the operator $\left(x \frac{d}{d x}\right)^{m}(m \in \mathbb{N})$ to both sides of (21) and set $s=0$, we can obtain a formula for the sum:

$$
\sum_{k=0}^{n} \frac{k^{m}}{\left(\begin{array}{l}
n \\
k
\end{array}\right)}
$$

Example 20. Setting $s=-\frac{1}{2}$ in (20), we obtain for $n \in \mathbb{N}_{0}$

$$
\sum_{k=0}^{n} \frac{(-1)^{k}\left(\begin{array}{c}
2 k \\
k
\end{array}\right)}{4^{k}\left(\begin{array}{l}
n \\
k
\end{array}\right)}=\frac{2(n+1) 4^{n}+(-1)^{n}(2 n+1)\left(\begin{array}{c}
2 n \\
n
\end{array}\right)}{4^{n}(2 n+3)}
$$

Example 21. Setting $x=\frac{1}{4}$ in Corollary 2, for any non-negative integer $n$, we have

$$
\sum_{k=0}^{n} \frac{\left(\begin{array}{c}
2 k \\
k
\end{array}\right)}{\left(\begin{array}{c}
n \\
k
\end{array}\right)}=\frac{(2 n+1)\left(\begin{array}{c}
2 n \\
n
\end{array}\right)}{5^{n+1}}\left(\sum_{k=0}^{n} \frac{5^{k}}{(2 k+1)\left(\begin{array}{c}
2 k \\
k
\end{array}\right)}+4 \sum_{k=0}^{n} \frac{5^{k}}{2 k+1}\right) .
$$

Example 22. Setting $x=-\frac{1}{4}$ in Corollary 2, for any non-negative integer $n$, we have

$$
\sum_{k=0}^{n} \frac{(-1)^{k}\left(\begin{array}{c}
2 k \\
k
\end{array}\right)}{\left(\begin{array}{c}
n \\
k
\end{array}\right)}=\frac{(2 n+1)\left(\begin{array}{c}
2 n \\
n
\end{array}\right)}{3^{n+1}}\left(4 \sum_{k=0}^{n} \frac{(-3)^{k}}{2 k+1}-\sum_{k=0}^{n} \frac{3^{k}}{(2 k+1)\left(\begin{array}{c}
2 k \\
k
\end{array}\right)}\right)
$$


Example 23. Let $n$ be a non-negative integer. Then,

$$
\sum_{k=1}^{n} \frac{F_{n+k}}{k\left(\begin{array}{l}
n \\
k
\end{array}\right)}=\sum_{k=1}^{n} \frac{F_{k-1}+F_{2 k-1}}{k},
$$

and

$$
\sum_{k=1}^{n} \frac{L_{n+k}}{k\left(\begin{array}{l}
n \\
k
\end{array}\right)}=\sum_{k=1}^{n} \frac{L_{k-1}+L_{2 k-1}}{k}
$$

Proof. Multiply both sides of (23) by $x^{2}\left(\frac{x+1}{x}\right)^{n}$ and then evaluate the resulting equation at $x=\alpha=\frac{1+\sqrt{5}}{2}$ and $x=\beta=\frac{1-\sqrt{5}}{2}$. This gives

$$
\sum_{k=0}^{n} \frac{\alpha^{n+k+2}}{\left(\begin{array}{l}
n \\
k
\end{array}\right)}=(n+1)\left(\sum_{k=0}^{n} \frac{\alpha^{2 k+1}}{k+1}+\sum_{k=0}^{n} \frac{\alpha^{k}}{k+1}\right)
$$

and

$$
\sum_{k=0}^{n} \frac{\beta^{n+k+2}}{\left(\begin{array}{l}
n \\
k
\end{array}\right)}=(n+1)\left(\sum_{k=0}^{n} \frac{\beta^{2 k+1}}{k+1}+\sum_{k=0}^{n} \frac{\beta^{k}}{k+1}\right) .
$$

If we divide both sides of these two equations by $n+1$ and then use (11), we find

$$
\sum_{k=0}^{n} \frac{\alpha^{n+k+2}}{(k+1)\left(\begin{array}{c}
n+1 \\
k+1
\end{array}\right)}=\sum_{k=0}^{n} \frac{\alpha^{2 k+1}}{k+1}+\sum_{k=0}^{n} \frac{\alpha^{k}}{k+1},
$$

and

$$
\sum_{k=0}^{n} \frac{\beta^{n+k+2}}{(k+1)\left(\begin{array}{c}
n+1 \\
k+1
\end{array}\right)}=\sum_{k=0}^{n} \frac{\beta^{2 k+1}}{k+1}+\sum_{k=0}^{n} \frac{\beta^{k}}{k+1},
$$

Letting $k+1=k^{\prime}$ (and $k^{\prime}=k$ ) and replacing $n$ by $n-1$, we obtain

$$
\sum_{k=1}^{n} \frac{\alpha^{n+k}}{k\left(\begin{array}{l}
n \\
k
\end{array}\right)}=\sum_{k=1}^{n} \frac{\alpha^{2 k-1}}{k}+\sum_{k=1}^{n} \frac{\alpha^{k-1}}{k},
$$

and

$$
\sum_{k=1}^{n} \frac{\beta^{n+k}}{k\left(\begin{array}{l}
n \\
k
\end{array}\right)}=\sum_{k=1}^{n} \frac{\beta^{2 k-1}}{k}+\sum_{k=1}^{n} \frac{\beta^{k-1}}{k}
$$

Now, the proof immediately follows from combining the Equations (47) and (48) according to the Binnet's formulas for $F_{n}$ and $L_{n}$. In this example, we can see the symmetrical properties of the Fibonacci and Lucas numbers which may be further exploited to obtain other finite sum identities.

\section{Concluding Remarks}

We have considered a generalization of a finite sum with parameters and analyzed its numerous consequences in relation to the representation of new identities. We have featured some identities involving central binomial coefficients, harmonic numbers, and Fibonacci and Lucas numbers. It is envisaged that further work could be undertaken involving a study of Cauchy numbers, Apery's constant, and other special numbers.

Author Contributions: Writing—original draft, N.B.; writing—review and editing, A.S. and N.B. All authors have read and agreed to the published version of the manuscript. 
Funding: No funding was sought or granted for this research.

Institutional Review Board Statement: Not applicable.

Informed Consent Statement: Not applicable.

Data Availability Statement: Not applicable.

Acknowledgments: The authors would like to thank the reviewers for their valuable comments and suggestions.

Conflicts of Interest: The authors have no conflict of interest.

\section{References}

1. Rockett, M.A. Sums of the inverses of binomial coefficients. Fib. Quart. 1981, 19, 433-445.

2. Sprugnoli, R. Sums of reciprocals of the central binomial coefficients. Integers 2006, 6, \#A27.

3. Gould, H.W. Combinatorial Identities, revised edition; West Virginia University: Morgantown, WV, USA, 1972.

4. Staver, T.B. Om summasjon av potenser av binomial koeffisienten. Nor. Mat. Tidsskr. 1947, 29, 97-103.

5. Pla, J. The sum of inverses of binomial coefficients revisited. Fib. Quart. 1997, 35, 342-345.

6. Trif, T. Combinatorial sums and series involving the inverses of binomial coefficients. Fib. Quart. 2000, 38, 79-84.

7. Sury, B. Sum of the reciprocals of the binomial coefficients. Eur. J. Combin. 1993, 14, 351-353. [CrossRef]

8. Mansour, T. Combinatorial identities and inverse binomial coefficients. Adv. Appl. Math. 2002, 28, 196-202. [CrossRef]

9. Sury, B.; Wang, T.; Zhao, F.Z. Some identities involving reciprocals of binomial coefficient. J. Integer Seq. $2004,7,3$.

10. Jin, H.T.; Du, D.K. Abel's lemma and identities on harmonic numbers. Integers 2015, 15, 2.

11. Batır, N. Combinatorial identities involving harmonic numbers. Integers 2020, 20, 2.

12. Graham, R.L.; Knuth, D.E.; Patashnik, O. Concrete Mathematics, 2nd ed.; Addison-Wesley: New York, NY, USA, 1994.

13. Belbachir, H.; Rahmani, M. Alternating sums of the reciprocals of binomial coefficients. J. Integer Seq. 2012, 15, 3.

14. Witula, R. Finite sums connected with the inverses of central binomial numbers and Catalan numbers. Assian-Eur. J. Math. 2008, 1, 439-448. [CrossRef]

15. Witula, R.; Slota, D.; Watlak, J.; Chmielowska, A.; Rózański, M. Matrix methods in evaluation of integrals. J. Appl. Math. Comput. Mech. 2020, 19, 103-112. [CrossRef]

16. Duren, P. Invitation to Classical Analysis; American Mathematical Society: Providence, RI, USA, 2012.

17. Srivastava, H.M.; Choi, J. Zeta and q-Zeta Functions and Associated Series and Integrals; Elsevier: Waltham, MA, USA, 2012.

18. Campbell, J.M. Identities for finite sums involving central binomial coefficients and harmonic-type numbers. Unpublished work, 2020.

19. Sofo, A. New classes of harmonic number identities. J. Integer Seq. 2012, 15, 12.

20. Sofo, A.; Cvijović, D. Extensions of Euler harmonic sums. Appl. Anal. Discrete Math. 2012, 6, 317-328. [CrossRef]

21. Sofo, A. Shifted harmonic sums of order two. Commun. Korean Math. Soc. 2014, 29, 239-255. [CrossRef]

22. Batır, N. Finite binomial sum identities with harmonic numbers. J. Integer Seq. 2021, $24,3$.

23. Sprugnoli, R. Alternating weighted sums of inverse of binomial coefficients. J. Integer Seq. 2012, 15, 3.

24. Belbachir, H.; Rahmani, M. Sury, Sums involving moments of reciprocals of binomial coefficients. J. Integer Seq. 2011, 14, 16. 